THE IMPORTANCE OF STUDYING DEVELOPMENTAL CHANGES IN COMPARATIVE PHYSIOLOGY AND BIOCHEMISTRY.

Warren W. Burggren.

University of Massachusetts, Amherst, Mass., U.S.A.

Many comparative physiologists and biochemists perceive that physiological complexity increases with animal development, and that consequent1y the adults are physiologically more complex than less mature stages. Consequently, the vast majority of studies in comparative physiology have been directed exclusively to investigation of the mature adult form of the species, with the expectation that studying the adults will give the greatest insights into questions of adaptation and evolution. This lecture will suggest that ontogenetic studies should be included in studies of physiological adaptation and its evolution, for several reasons. 1) Natural selection, which is the basis of environmental adaptation and evolution, acts on all developmental stages, not just adults. 2) Small individuals of a species differ

physiologically from larger individuals due to allometry (scaling). Thus, immature individuals may respond to an environmental challenge quite differently from adults simply by virtue of their size difference. 3) Animals frequently undergo changes in habitat and lifestyle during the course of development (confined in egg or uterus to free-living; water breathing to air breathing; aquatic to terrestrial). Such changes inevitably result in physiological differences between early developmental stages and adults, and of ten the physiology and biochemistry of the transitional stages is more complex rather than less complex when compared with adults. Theoretical arguments based on evolutionary theory, as well as case studies of development of cardiorespiratory systems, will be used to argue that a thorough understanding of the physiology of a species requires that a wide developmental range of individuals be investigated, rather than the typical approach of focusing on a single stage or very limited range of developmental stages. 
Comparative pharmacology of neurotransmitters and neuromodulators. Faculty of Integrated Arts and Sciences, Hiroshima Univ.

Over the last thirty years, pharmacological techniques developed in medical science field have been applied to the study of invertebrate nervous systems, and comparative neuropharmacology has emerged as a field of neurobiology. The aim of comparative neuropharmacology is to clarify neural mechanisms from the biological view point by using pharmacological techniques. In this field, comparative study of the actions of not only drugs but also neurotransmitters and neuromodulators, which can be regarded as endogenous drugs, is of very importance.

In general, the non-peptidic neurotransmitter species (classical neurotransmitter species such as acetylcholine and serotonin) of vertebrates are also utilized in invertebrates. However, the pharmacological properties of their receptors in invertebrates are not identical with those in vertebrates. In some molluscs, for instance, eserine has been suggested not only to inhibit acetylcholinesterase activity but also to block acetylcholine receptors. Strychnine, which is well known as a glycine-receptor blocker in vertebrates, has also been shown to block acetylcholine receptors in some molluscan neurones and muscles. Therefore, we should be very careful when we use neurotropic drugs, which have been developed in medical science field, for the study of neural mechanisms of invertebrates.

In contrast to the case of the classical neurotransmitters, there seems to be no neuropeptide species which is utilized in both vertebrate and invertebrate, though it is known that there are a number of invertebrate peptides whose amino acid sequences are significantly homologous with those of vertebrate peptides. Furthermore, we can point out the following interesting matters as comparative aspects of invertebrate neuropeptides; (1) peptides structurally related to each other are often found to be distributed interphyletically, (2) it is not rare that more than ten analogue peptides, which belong to a family, are present in an animal species, (3) it is also not rare that a fragment peptide shows more potent aotion than the fullstructure peptide, (4) a number of neuropeptides having D-type amino acid residue (s) may be present, (5) it appears that many species of neuropeptides are, in general, involved in the regulation of contraction of a muscle, and (6) it is considered that a large part of invertebrate neuropeptide species are remained to be identified. 
Comparative Physiology and Biochemistry of Signal Transduction - Introductory talk Motoyuki Tsuda Department of Life Science, Himeji Institute of Technology, Shosha 2167, Himej1 671-22. Japan

Signal transduction in the cells is one of the most important and widely studied area in the life science. There is a striking similarity between the biological pathway of interacellar signaliing by light and those by hormones or neurotransmitters and all the signal transduction system appear to have a simillar molecular basis action. Recent progress in recombinant DNA technique provide cDNA sequence information for a number of component involved signal transduction in different cells. These studies demonstrated gross structural and topological similarities in the receptor and transducer molecules and comprise a large families of genes( rhodopsin supergene family or G-protein coupled supergene family)derived from an ancestral precursor by gene duplication.

In this symposium, signal transduction systems of different organs from animals, plant and microbiology will be presented. Signal transduction and desensitization between muscarinic acetylcholine receptor and G-protein in brain system(by Dr. Haga)were appeared to be similar to those of visual system(by Dr. Iwasa). Cyclic nucleotide regulated ion channel, which was formerly found in vertebrate photoreceptors, were also found in taste receptor cells(by Dr. Tonosaki). Recently, the components of these signal transduction were also found in michrobiology and plant. Rhodopsin-like pigments in archbacteria appears to be receptor for phototaxisis for these bacteria(by Dr. Mukohata). G-proteins from plant were characterized by bacteriotoxin(by Dr. Hasunuma).

Signal transduction in the brain: Interaction of muscarinic acetylcholine receptors with G proteins and receptor kinase. Tatsuya Haga, Kazumasa Shiozaki, Kazuko Haga. Department of Biochemistry, Institute of Brain Research, Faculty of Medicine, University of Tokyo, Hongo, Tokyo

Muscarinic acetylcholine receptors were purified from porcine atrium and reconstituted with $G$ proteins ( $G i$ and Go) purified from porcine cerebrum into crude lipid vesicles. Approximately $70 \%$ of reconstituted receptors showed GTP- or GDPsensitive high affinity agonist binding irrespective of the presence or absence of $\mathrm{Mg}$ and $30 \%$ of receptors showed low affinity. Apparent dissociation constants for carbamylcholine binding to the high affinity sites in the presence of $10 \mathrm{mM} \mathrm{Mg}$ or 40 $\mathrm{nM} \mathrm{Mg}$ and to the low affinity site in the presence of GTP or GDP were $0.27,13$ and $160 \mu \mathrm{M}$, respectively. Apparent affinity of reconstituted g proteins for GDP but not for GTP decreased in the presence of both carbamylcholine and $\mathrm{Mg}$ but not in their absence. These results suggest that there are two kinds of agonist-receptor-G protein complexes and that the presence of $\mathrm{Mg}$ is required for the formation of the ternary complex with a high affinity for agonists and a low affinity for GDP. Cerebral but not atrial receptors were phosphorylated by protein kinase $\mathrm{C}$ and atrial receptors were phosphorylated by cAMP-dependent protein kinase. These phosphorylations have not been affected by the presence or absence of muscarinic agonists. The protein kinase which phosphorylates muscarinic receptors depending on the presence of agonist was partially purified from an extract of porcine brain. Muscarinic receptors purified either from porcine cerebrum or atrium were phosphorylated only in the presence of carbamylcholine, and the effect of $1 \mathrm{mM}$ carbamylcholine was antagonized by $10^{-5} \mathrm{M}$ atropine. The activity was observed in the presence of EGTA and EDTA, and the presence of Ca $(0.5 \mathrm{mM}$ in a free form) suppressed the activity slightly. The activity was not affected by $0.1 \mathrm{mM}$ cAMP or cGMP and was inhibited by $0.15 \mathrm{M} \mathrm{NaCl}$ and $\mathrm{KCl}$. This activity appears to be due to $\beta$ adrenergic receptor kinase itself or a similar kinase. G proteins have dual effects on the agonist-dependent phosphorylation of muscarinic receptors, i.e. stimulation at lower concentrations and inhibition at higher concentrations. The stimulatory effect was reproduced with the $\beta r$ subunit of $G$ protein and the inhibitory effect with the combination of the $\alpha$ and $\beta \gamma$ subunits.

Vol. 7 , No. 4 (1990) 
Taste transduction mechanism of the mouse taste cell.

Keiichi TONOSAKI Dept. of Oral Physiology, Sch. of Dentistry, Asahi Univ.

Taste receptor cell excitation begins with the adsorption of a taste stimulus by taste receptor membrane. Taste receptor cells are elongate neuroepithelial cells located in taste buds. Taste cells are small cells embedded in a tough, water-proof epithelium. A typical taste bud (diameter about $50 u m$ ) contains about 20 or more receptor cells, each with a tiny portion of its membrane exposed in a taste pore (diameter about 1-2um in the mammal) on the mucosal surface of the tongue. Tight junctions found at the apical membrane of taste cells do not allow stimuli to enter the taste bud, making it difficult to alter the environment of the taste receptor cell by superfusing with chemical solutions. Insertion of glass microelectrode into the taste bud anywhere other than through the taste pore seems impossible. These features render them difficult to impale with intracellular microelectrode and difficult to preserve with conventional biochemical experiments. Thus, a few researchers have studied mammalian taste responses with intracellular recording techniques. Studies of intracellular recordings of taste receptor cell responses to taste stimuli suggest that different transduction mechanisms may underlie each of the primary taste sensations. It is already demonstrated that c-GMP induces a membrane depolarization accompanied by a membrane resistance increase. The results suggest that when sucrose adsorbs to the taste receptor site, the internal c-GMP concentration is increased. An increase in C-GMP concentration leads to a decrease in potassium conductance. This hypothesis is supported by the effects of TEA injections into the cell and by the voltage-clamp results. When the outward current was stopped (or decreased), the decreased outward current is reflected on the result of the inward direction of current flow. It has 2 also been reported that bitter substances cause a rise in the intracellular $\mathrm{Ca}^{2+}$ concentration due to release from internal stores in the taste cell, leading to the speculation that the release of $\mathrm{Ca}^{2+}$ from internal stores implicated the involvement of the IP 3 system in bitter taste transduction. However, very little is known about the molecular mechanisms involved in the taste transduction processes.

Comparative study of signal transduction process in visual cells

Tatsuo IWASA, Dep. Life Sci., Fac. Sci., Himeji Institute of Technology

A transduction cascade triggered by absorption of photon results an electrical signal in the photoreceptor cells. The process in higher vertebrates have been extensively studied and is one of the most well-known systems in signal transduction systems. It composed of photoreceptor protein rhodopsin (Rh), G-protein; transducin (Gt), c-GMP phosphodiesterase (PDE), c-GMP dependent channel, rhodopsin kinase, and arrestin (Ar). On the system in invertebrate, however, much less is known; proteins coupled with $\mathrm{Rh}$ and ion channel are not isolated yet. Recent progress in the signal transduction in invertebrate visual cells is summalized and compared with that in vertebrate.

Rhodopsin: Rhodopsin is a highly conserved protein and one of the members of "rhododopsin super gene family" characterized by their topology in the membrane; they span the membrane seven times. The complete coding region of the Rh of the most primitive species, lamprey, was obtained by PCR methods, showing 78-82\% similarities to Rhs of higher vertebrates. The red color pigment gene was isolated from chicken. The amino acid sequences of invertebrate Rhs were known only in octopus and Drosophila. G-protein: In octopus, the light induced GTPase activity was found and the caracterization by bacterial toxins showed that it is rather homologous to those in hormone system than that in vertebrate visual system, Gt. Its $\alpha$-subunit showed apparent molecular weight of 41 kDa and named as Gip. Rhodopsin kinase: In vertebrate visual cells Rh kinase and arrestin are involved in the desensitization process. Octopus rhodopsin is also phosphorylated after an irradiation of the light. The degree of phosphorylation was much more dependent on cAMP than cGMP, suggesting that Rh kinase of octopus is cAMP-dependent kinase like in the other signal transduction systems.

The studies on octopus visual cells indicate that the transduction system of octopus visual cells is much more general one than in vertebrate system. The further studies in the invertebrate visual systems are necessary. 
Photoreceptors in Archaebacteria

Yasuo MUKOHATA Department of Biology, Faculty of Science, Nagoya University

In most of extremely halophilic archaebacteria retinoid proteins are expressed to respond to light. Some of them are ion pumps coupled to energy transduction of the bacteria and others are sensors which transmit the light information to flagellar motors. In Halobacterium salinarium (halobium) bacteriorhodopsin (bR, $\mathrm{H}^{+} \mathrm{pump}$ ) halorhodopsin ( $h R, \mathrm{Cl}^{-}$pump), sensory rhodopsin ( $\mathrm{sR}$, photosensor) and phoborhodopsin ( $\mathrm{pR}$, photosensor appearing only in the log phase) are found. In Halobacterium sp. collected in Western Australia, $\mathrm{H}^{+}$pumps different from bR are found to be archaerhodopsins (aR's). In Natronobacterium pharaonis, a $\mathrm{Cl}^{-}$pump different from hR is found to be pharaonis $h R(p h R)$. The amino acid sequences deduced from the cloned genes of bR, hR, sR, aR, aR-2 and phR postulate that all of them are one (halo)bacterial retinoid protein family.

One of the two key aspartic acid residues in $\mathrm{bR} \mathrm{H}^{+}$pump is missing in sR, photosensor, which is thus unable to pump $\mathrm{H}^{+}$across the membrane in the light. For transduction of light signals, sR should change its conformation, which will trigger and eventually control flagellar motors. The intermediary messenger may be protein methylation and/or fumarate. Such a conformation change by light is found in bR, which includes sliding in the axial direction and/or tilting of the helices.

From the sequence analysis of halobacterial ATPase, it is suggested that some primitive archaebacteria are the host in the endosynbiotic evolution of eukaryotes. The archaebacterial retinoid proteins and the retinoid proteins and their relatives in eukaryotes would have the same origin. There are afew amino acid residues still conserved among halobacterial and eukaryotic retinoid proteins, especially in the putative helix regions.

Light signal transduction in etiolated pea seedings Kohji Hasunuma Yokohama City Univ., Kihara Inst. Biol. Res.

Winslow R. Briggs Carnegie Inst. Mashington, Dept. Plant Biol.

Third internodes of etiolated pea seedings are known to be highly sensitive to light. Third internodes were excised and irradiated with red light $\left(0.25 \mathrm{mmole} / \mathrm{m}^{2} /\right.$ sec) for 20,40 , or 60 sec or irradiated for 40 sec with various intensity from $0.04,0.12$ to $0.25 \mathrm{mmole} / \mathrm{m}^{2} / \mathrm{sec}$. As a dark control, $40 \mathrm{sec}$ of irradiation with dim green safelight was performed. Immediately after red light irradiation the samples were ground and squeezed with nylon cloth. Plasma membrane was purified by two diferential centrifugations followed by sequential phase partition. We devised a native gel electrophoresis by use of $1 \%$ Triton $X-100$. After binding ( $\alpha-32$ P)ATP or $(\alpha-32$ P)GTP to ATP-GTP-binding proteins in plasma membrane, the samples were analyzed by Triton X-100-PAGE. At least 10 species of ATP-GTP-binding proteins were detected and designated as AGP-1 to -10. Nucleotide specificities of these ATP-GTP-binding proteins in plasma membrane were examined by use of ( $\alpha-32 P)$ ATP or $\left(\alpha-32\right.$ P)GTP with $10^{-5}$ or $10^{-4}$ of ATP, GTP, CTP or UTP. AGP-1 and AGP-2 showed strong specificity to ATP and weak specificity to GTP. AGP-3, $-4,-5$, and -6 showed strong specificity to ATP by use of $(\alpha-32 P) A T P$ and weak specificity to GTP. By use of ( $\alpha-32 P)$ GTP, however, they showed strict specificity to GTP. AGP-7 and -8 bound $(\alpha-32 \mathrm{P})$ ATP and $(\alpha-32 \mathrm{P})$ GTP.

By the red light irradiation of stem section, 2 3-fold stimulation of the binding of $(\alpha-32$ P)ATP to AGP-1, $-2,-3,-4,-7$ and -8 was observed. Similar stimulation of the binding of $(\alpha-32 P)$ GTP was observed in AGP-3 and -4 . Plasma membranes purified from these red light irradiated samples were subjected to SDSPAGE. Western blotting by use of antibodies for $\alpha$-subunit of bovine transducin ( $\mathrm{T} \alpha$ ) and for $\beta, \gamma$-subunit of transducin (T $\beta, \gamma$ ) revealed that protein bands with $\mathrm{Mr}$. $37 \mathrm{KD}$ and $125 \mathrm{KD}$ cross reacting to anti-T $\alpha$-antibody faded out by the red light irradiation. Protein bands ith $M r .30 \mathrm{KD}$ and $40 \mathrm{KD}$, cross reacting to antibody for $T \beta, r$, however, showed no change. AGP-1 was identified to be 37 KD protein. (32P)ADP-ribosylation of these samples by pertussis toxin revealed that 37 $K D$ protein was ( $32 P$ ) ADP-ribosylated with transient stimulation by red light irradiation, in vivo.

Vol. 7 , No. 4 (1990) 
Target dependence of motoneuronal survival. Yasuhiro KASHIHARA National Institute for Physiological Sciences, Okazaki

At early embryonic stage, massive neuron death occurs in spinal cord during normal development. These stages coincide with those at which motoneurons normally form functional connections with the muscle. When the limb bud is removed before the formation of neuromuscular connections, all motoneurons eventually die. Thus, the survival of motoneurons appears to depend on their target.

Is the target dependence of motoneuron survival previously observed at embryonic stage still present during the early post-natal period? However, in the investigation on reinnervation of muscle in neonatal rat, no sign of motoneuron death has been noticed following section of a nerve near the muscle. We have examined whether motoneuron death can be induced if the cut nerve is prevented from reinnervation of the muscle in neonatal rats. The results show that target dependence of motoneuron survival is still present during the early post-natal life, as is the case at embrionic stage.

Programmed cell death in the nematode Caenorhabditis elegans. RYUJI HOSONO Dept. Biochem., Sch. Med., Kanazawa Univ.

In C. elegans, 671 cells are generated during embryogenesis and 419 cells are added during postembronic development. In the course of the lineage, 231 cells subsequently die. Their identity and the approximate times of their deaths are predictable. Four genes, ced-1, ced-2, ced-3 and ced-4 contributing to the programmed cell death are identified. From the genetic studies, the process of the cell death are dissected into three steps : intiation, engulfment and DNA degradation. In several ced mutants, almost cells survive and become neuronal cells. However, these animals behave normally. 
Posteclosional cell death in the nervous and muscular system of Drosophila melanogaster

Ken-ichi KIMURA Lab. of Biol., Hokkaido University of Education, Iwamizawa Campus

Programmed cell death is a fundamental feature of developing nervous system of multicellular organism. The cell death is thought to be programmed under the interaction of extrinsic factors and a series of intrinsic genetic control. Here $I$ will show the extrinsic factors of the cell death in the nervous and muscular system of Drosophila melanogaster and the trial to identify the genes involved in the programmed cell death.

Programmed cell death occurs in the nervous and muscular system of a newly eclosed fly, Drosophila melanogaster. Many of the abdominal muscles which were used for eclosion behavior breakdown by $12 \mathrm{hr}$ after eclosion. Related neurons in the ventral ganglion also die within $24 \mathrm{hr}$. Ligation of eclosing flies at the neck has no effect on the muscle degeneration, however it prevents the neuronal death. These results show that control over the muscle death differs from that of the neuronal death. The muscle death is triggered by a signal which comes from the anterior region, presumably the head, just before eclosion. In contrast to the muscle death, the neuronal death is triggered by the signal which is closely correlated with the initiation of wing spreading behavior after eclosion.

An administration of actinomycin $D$, which is an inhibitor of RNA synthesis, prevented or delayed the abdominal muscle death after eclosion. This indicates that the cell death is an active process that requires the activation of new genetic information. We are trying to identify the genes involved in the cell death by means of screening the mutants which cause the defect in the muscle death and screening enhancer-trap lines. Two mutants which delay the muscle death in the head after eclosion were isolated. They also showed the defect in the cell death in the abdominal muscles. Also one line which was isolated from the enhancer-trap lines showed the beta-galactosidase expression in the doomed muscles of the abdomen. 
Control of circulation by hormonal secretion: in the case of pericardial organs of decapod crustaceans. Taketeru KURAMOTO Inst. Biol. Sci., Tsukuba Univ.

In general, blood circulation is controled by both centra1 nervous and endocrine systems via excitatory and inhibitory media. This principle was applied to that of decapod crustaceans. The dorsal nerves of thoracic gang1ion quick1y controlled each of restrictive parts; nerve and muscle cells in the heart and arterial valves. Their excitatory and inhibitory transmitters seemed to be dopamine (DA) and gammaamino butyric acid (GABA), respectively. Whereas, pericardial hormones, serotonin ( $5-H T)$, octopamine (OA), dopamine (DA), proctolin (Pr) and crustacean cardioactive peptide (CCAP), appeared to act slowly on the heart for minute-1ong periods. Moreover, the targets and actions of these hormones were multiple and versatile (see Table). In lobsters, the main targets were the sma 11 cardiac neurons, the cardiac muscle and the flap muscle in cardioarterial valves. Further, the secretory cells themselves or their higher center might be included in the targets. After the actions on these targets were integrated as a whole in the ganglion, the rate and amplitude of heartbeat was determined including the direct action on muscle contraction. 'Experiments in situ and in vivo suggests that $O A$ and $P r$ may be released during food chewing and enhance the heart pump. Furthermore, ligament nerves (pericardial organs of Panuli rus) fired as soon as temperature of perfusate was lowered, even if the drop was $0.5-1^{\circ} \mathrm{C}$. Therefore, circulation of decapods may be altered with a smal1 change in temperature of the body and/or environment.

\begin{tabular}{|c|c|c|c|c|}
\hline Targets $\begin{array}{c}\text { Origin } \\
\text { Media }\end{array}$ & $\begin{array}{l}\text { Pericardial } \\
O A \text { S-HT }\end{array}$ & $\begin{array}{l}\text { Orga } \\
\text { DA }\end{array}$ & & CCAP \\
\hline \multicolumn{5}{|l|}{$\begin{array}{l}\text { Cardiac Ganglion } \\
\text { Swall Cells }\end{array}$} \\
\hline & & + & $(+)$ & $?$ \\
\hline Burst Duration & --++ & + & $(+)$ & $?$ \\
\hline \\
\hline Burst Rate & +--+ & + & $(+)$ & $?$ \\
\hline Burst Duration & $+-\quad+$ & + & $(+)$ & $?$ \\
\hline \\
\hline & -++- & + & + & ? \\
\hline Tension & $+\quad+-$ & + & ++ & $?$ \\
\hline \multicolumn{5}{|l|}{$C-A$ Valves } \\
\hline Anterior Valves & $-(-+)$ & + & ++ & $?$ \\
\hline Posterfor Valve & $+(-+)$ & + & ++ & $?$ \\
\hline $\begin{array}{l}\text { Beat Rate } \\
\text { Beat Anplitude }\end{array}$ & $\begin{array}{c}-++ \\
+++\end{array}$ & $\begin{array}{l}+ \\
+\end{array}$ & $\begin{array}{c}+ \\
++\end{array}$ & $\begin{array}{l}++ \\
++\end{array}$ \\
\hline & & & & \\
\hline SSC & -+++ & - & ++ & $?$ \\
\hline
\end{tabular}

,+++ and,$---:$ increase and decrease in function 1 ith grade, respectively. +-., + ; small or biphasic changes. ( ): suspect matter. ?: unknown matter. C-A; cardio-arterial. FSC. SSC: first, second systolic contraction.

Functional development of the cardio-inhibitory nerve in juvenile stage of the isopod crustacean, Ligia exotica. Hiroshi YAMAGISHI, Inst. of Biol. Sci., Univ. of Tsukuba

The neurogenic heart of the isopod crustacean Ligia exotica is innervated by a pair of the cardio-regulatory nerve, each of which contains one inhibitory and two accerelatory nerve fibers. The inhibitory nerve makes inhibitory synaptic cotacts with both the cardiac ganglion cells and myocardial cells. The neurogenic heart beat decreased in frequency, amplitude and tonus with increasing stimulus frequency of the inhibitory nerve. In a silent heart, tonus of the heart decreased according to increase in frequency of the inhibitory stimulation. These results seem to indicate that the peripheral innervation of the cardio-inhibitory nerve contribute to regulation of the heart tonus.

However, the heart muscle of Ligia has been reported to have latent alltomatic nature. When spontaneous activity of the cardiac ganglion was completely stopped

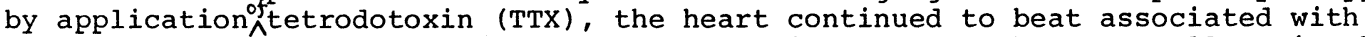
spontaneous activity of the heart muscle. This suggests that, as well as in the cardiac ganglion, the cardio-inhibitory nerve supresses the generation of spontaneous activity in the heart muscle.

In order to elucidate physiological role of the latent myogenicity of the heart, the heart beat of juveniles just released from the brood chamber of a parent was examined. Electophysiological investigation revealed that the heart beat of Ligia juveniles is myogenic. Repetitive stimulation of the cardio-inhibitory nerve produced inhibitory junctional potentials (IJPs) in the heart muscle and supressed the myogenic heart beat. In one to two weeks after leaving the parent, the heart beat of the juveniles became neurogenic. Repetitive stimulation of the inhibitory nerve supressed the neurogenic heart beat. These results indicate clearly that the inhibitory neural regulation of the heart muscle preceds ontogenically that of the cardiac ganglion. 
Physiological and biochemical studies of the cardioregulatory mechanisms in molluscs. Mariko Fujiwara-Sakata Physiol. Lab., Fac. Int. Arts and Sci., Hiroshima Univ.

It has been known that the molluscan heart is myogenic and the rhythm of the heart beat is controlled by several neuropeptides in addition to some classical transmitters such as 5-hydroxytryptamine(5-HT) and acetylcholine(ACh).

In the heart of a prosobranch, Rapana thomasiana, both 5-HT and FMRFamide (H-PheMet-Arg-Phe- $\mathrm{NH}_{2}$ ) exhibited excitatory effects, and ACh and CARP(Catch relaxing peptide; H-Ala-Met-Pro-Met-Leu-Arg-Leu- $\mathrm{NH}_{2}$ ) showed inhibitory ones. FMRFamide had greater inotropic and choronotropic effects than 5-HT. The effects of 5-HT, and none of those of FMRFamide and of stimulating the excitatory cardiac nerves, were blocked by methysergide. CARP markedly inhibited both the amplitude and frequency of the heart beat and the threshold was lower than that of ACh. The effects of ACh and of stimulating the inhibitory cardiac nerves were blocked by benzoquinonium. while those of CARP was not blocked. By the immunohistochemical study, it was found that the atrium includes both FMRFamide- and CARP-like materials, but the ventricle does not. The visceral ganglia also had many FMRFamide- and CARP-like substances.

In a pulmonate, Achatina fulica, application of 5-HT as well as stimulation of a heart excitatory neuron, PON, resulted in the enhancement of the heart beat. The enhancement was blocked by methysergide. FMRFamide also enhanced the beat of the ventricle and potentiated the excitatory response of the atrium to both PON stimulation and 5-HT application. Achatina cardio excitatory peptide- 1(ACEP-1; H-Ser-Gly-Gln-Ser-Trp-Arg-Pro-Gln-Gly-Arg-Phe- $\mathrm{NH}_{2}$ ) isolated from the atria of this snail showed the remarkable excitatory effect on the ventricle. The atrium and the aortic region of the ventricle included both FMRFamide- and ACEP-1-1ike materials. In the subesophageal ganglia, there were many cells containing FMRFamide- and ACEP-1-like substances. Although the mechanisms of actions of the peptides are still not yet clarifled, physiological and immunohistochemical findings presented here show the possibilities that these peptides play physlological roles as cardioregulatory substances.

Central and peripheral neuronal mechanisms in the Aplysia gill. Makoto Kurokawa and Kiyoaki Kuwasawa Dept. Biol., Fac. Sci., Tokyo Metropolitan Univ.

In the circulatory system of Aplysia, movements of the gill located between the heart and haemocoel significantly affect the haemolymph flow to the heart. When the vascular muscle of the gill contracts in response to the cessation of arterial perfusion, even the haemolymph pressure of the artery is raised (Furgal \& Brownell, 1987). Several gill motor neurons have been identified in the abdominal ganglion in the central nervous system. The gill has the peripheral nervous system which involves three orders of neural element, i.e., peripheral nerves arising from the abdominal ganglion, the branchial ganglion'situated at the insertion of the branchial nerve in the gill, and neurons scattered in the neural plexus beyond the branchial ganglion. We studied relationship between the central and peripheral nervous systems in gill movements. Aminergic innervation of the gill, heart and artery was proved with glyoxylic acid-induced fluorescence and immunocytochemical techniques. Both the branchial ganglion and the neural plexus contain gill motor neurons. The motor neurons in the branchial ganglion received both excitatory and inhibitory sensory inputs from, respectively, tactile receptors and pressure proprioceptors. From results of HPLC analysis, we believe that the tactile receptors are dopaminergic. A certain identified motor neuron (analogous to L7 in A. californica) in the abdominal ganglion has the interneuronal function having collaterals to make excitatory synaptic contact with motor neurons in the branchial ganglion. We elucidated four types of inhibitory innervation. One of them is presynaptic inhibition at the terminal of the gill motor neurons contained in the abdominal ganglion. Serotonin may mediate the presynaptic inhibition. In the heart and artery, serotonin immunoreactive neural processes were observed. Serotonin produced excitatory effects on the heart. In the anterior artery serotonin produced longitudinal relaxation and rhythmic contraction. The results indicate that the overall cardiovascular system is under close control by monoaminergic nerves though actions of monoamines varying according to sites in the circulatory system have not yet been systematically understood from a haemodynamic viewpoint. 
Endothelins - A new family of regulatory peptides in mammalian vascular homeostasis. Masashi YANAGISAWA Inst. of Basic Med. Sci., Univ. of Tsukuba

Endothelins (ETs) are the newly discovered family of mammalian vasoactive peptides. The first member of the family, endothelin-1 (ET-1), was originally identified as a 21 -amino-acid potent vasoconstrictor peptide found in the culture supernatant from vascular endothelial cells. Subsequent studies have demonstrated three separate endothelinrelated genes in the human genome, which encode for three distinct isopeptides of the endothelin family, ET-1, ET-2 and ET-3, respectively. It is currently believed that many other mammalian species also produce three isopeptides with amino acid sequences identical or very similar to human isopeptides. Furthermore, peptides with striking structural and pharmacological similarities to mammalian endothelins, Sarafotoxins, are present in certain snake venoms and play roles as a major toxic component. Phylogenically, immunoreactivity related to mammalian endothelins has been reported even in some invertebrates. Molecular cloning of these three isopeptides in human has revealed that they are expressed in multiple tissues with distinct distribution patterns: while only ET-1 is detected in the vascular endothelium, ET-2 and/or ET-3 are expressed in other tissues such as the brain, lung, kidney, adrenal gland, intestine and placenta. ET-1 is also expressed in many extra-vascular tissues including the brain, kidney and lung.

Apart from their potent and long-lasting vasoconstrictor/pressor activities, endothelins possess a wide spectrum of both vascular and non-vascular actions in various tissues, including the airways, myocardium, renal tubules and glomeruli, adrenal cortex and medulla, gastrointestinal tract, fetoplacental system, and central and peripheral nervous system. In accordance with these observations, radioligand binding studies have demonstrated a wide tissue distribution of specific binding sites for endothelins. Furthermore, the existence of multiple (at least two) subtypes of endothelin receptors with different affinities to the three isopeptides has also been demonstrated.

In cultured endothelial cells, the production of ET-1 is regulated by various chemical and mechanical stimuli, such as thrombin, TGF- $\beta$, angiotensin II, vasopressin, and hemodynamical shear stress. Furthermore, our recent studies in isolated perfused rat mesenteric arteries have demonstrated an extremely slow vasoconstrictive response to several hours of continuous perfusion low concentrations of vasopressin, which seems to be mediated by endogenously de novo produced ET-1. The induction of ET- 1 by thrombin and TGF- $\beta$ implies that ET-1 may be involved in the maintenance of vascular constriction in primary hemostasis, in the repair process of vascular injury, and/or in the genesis of pathological thrombosis. The plasma levels of ET-1 in humans and experimental mammals have also been measured under various conditions by using highly sensitive and specific immunoassay. Those measurements have shown that ET-1 levels in peripheral plasma are significantly elevated after acute physical stresses, such as myocardial infarction, cardiogenic shock, major surgical operation, and challenge with bacterial endotoxin, suggesting the physiological and/or pathophysiological roles of endothelins in these processes.

Two barosensitive neuron groups in the ventrolateral medulla oblongata that control the vasomotor activity in rabbits. Naohito TERUI Institute of Basic Medical Sciences, University of Tsukuba, Tsukuba, Ibaraki 305, Japan

Activity of two neuron groups were recorded from the ventrolateral medulla of the urethane-anesthetized rabbit. Neurons of the first group, RVLM neurons, located in the rostral ventrolateral medulla (RVLM), received inhibitory inputs from the arterial baroreceptors, excitatory inputs from peripheral chemoreceptors and a mixture of excitatory and inhibitory inputs from both somatic and visceral afferents $1,2,3$. They sent their axons to the spinal cord. Since spontaneous and evoked activity of the RVLM neurons always preceded about 100 ms to those of peripheral vasomotor fibers (the renal nerve), they were seemed to be excitatory premotor neurons for the preganglionic vasomotor fibers. Neurons of the second group, CVLM neurons, located in the caudal ventrolateral medulla (CVLM), received excitatory inputs from the arterial baroreceptors and projected to the RVLM. The mean conduction time of their antidromic spikes from the RVLM was $10.5 \mathrm{~ms}$ and the mean onset latency of the excitatory response to stimulation of the baroreceptor afferent fibers was $37.5 \mathrm{~ms}$. The sum of these two values was very close to the mean onset latency of the inhibitory response $(47 \mathrm{~ms})$ of the RVLM neurons to stimulation of the baroreceptor afferent fibers ${ }^{4}$. Therefore, it is very likely that these CVLM neurons mediate the arterial baroreceptor reflex as interneurons between the nucleus tractus solitarius, where the arterial baroreceptor afferents terminate, and the RVLM. These CVLM neurons were confirmed to receive somatic inputs. It is plausible that the CVLM neurons and the RVLM neurons jointly integrate inputs from various sources and convey the sum of this information to the sympathetic preganglionic fibers through the axons of the RVLM neurons.

1. Terui, N., Saeki, Y., and Kumada, M. (1986) Jpn. J. Physiol. 36: 1141-1164.

2. Terui, N., Saeki, Y., and Kumada, M. (1987) Can. J. Physiol. Pharmacol. 65: 1584-1590.

3. Saeki, Y., Terui, N., and Kumada, M. (1988) Jpn. J. Physiol. 38: 267-281.

4. Terui, N., Saeki, Y., Masuda, N., and Kumada, M. (1990) Neurosci. Lett. (in press) 
A highly sensitive two-site enzyme immunoassay for calcitonin gene-related peptide. Masahiko SAKAGUCHI National Institute for Physiological Sciences, Okazaki

We have developed a highly sensitive two-site enzyme immunoassay for calcitonin gene-related peptide (CGRP), based on the sandwiching of CGRP between anti CGRP antibody IgG coated to a polystyrene bead and anti CGRP antibody Fab'-linked horseradish peroxidase (HRP). Detection of the fluorescent HRP reaction product permitted the determination of 1 pg of CGRP per assay. Using this assay, we quantified the amount of CGRP released from rat soleus muscle by the dorsal root nerve stimulation. The intensity of effective nerve stimuli suggested that the sensory fibers responsible for CGRP release are $A \delta$ and $C$ fibers.

Immunoelectron Microscopy to Study the Localization of Proteins.

Yohki HIEDA Dept. Mol. Biol., School of Sci., Nagoya Univ.

Immunohistochemical techniques have been recently used as powerful tools to study the localization of various proteins in tissues or cells as the improvement of the techniques. At light microscopic level we can examine tissue distribution and cellular localization of proteins using handy techniques such as immunofluorescent microscopy. Immunolabeling experiments in electron microscopy provide more precise information on the subcellular localization of proteins, while this technique has three problems to be overcome, that is, preservation of ultrastructure and of antigenicity, and accessibility of antibody to tissues or cells. Although some kinds of method are available in which antibody reaction is performed during the process for conventional electron microscopy, the most ideal and reliable is the method using ultrathin frozen sections. In this symposium, procedures of some methods for immunoelectron microscopy are described, taking as example membrane skeletal proteins. 
How to make use of the PCR in genetic engeneering laboratories

Kiyozo Asada Biotechnology Research Laboratories

Takara shuzo Co., Ltd.

The polymerase chain reaction (PCR), principle of which was first described by Saiki et al. in science in 1985, was established as a practical method by the introduction of thermostable DNA polymerase derived from Thermus aquaticus (Taq DNA polymerase). This technique is now used in thousands of laboratories in various fields including archaeology and forensic medicine. In this seminor, practical usage of the PCR in genetic engeneering laboratories can be discussed focusing on the following procedures.

\section{DNA cloning}

PCR can be used in DNA cloning, even if information is limiting to design couple of primers at the ends of a region to be cloned. Following the restriction enzyme digestion of DNA and adaptor ligation, target region can be amplified with one primer complementary to part of target sequence and the other complementary to adaptor sequence; thus target sequece can be cloned. We have so far cloned several genes by this method and one example can be presented.

\section{DNA sequencing}

We can avoid cultivaton of E.coli to prepare template DNA for dideoxy chain reaction for sequecing by the introduction of the PCR; amplified DNA can be directly used as a template (direct sequencing). So far, direct sequecing with single stranded DNA preapared by asymmetric PCR has been reported. But this method does not contantly works because of the difficulty of preparation of single stranded DNA. We have found that double stranded DNA prepared by the PCR can be used for sequencing by modifying protocoal generally used for super-coiled sequencing. Modified protocoal can be presented.

Analysis of receptor movements and assembly by using time-resolved fluorescence microscopy and nanometer-video enhanced contrast microscopy. Akihiro KUSUMI Department of Pure and Applied Sciences, College of Arts and Sciences, The University of Tokyo, Meguro-ku, Tokyo 153, Japan.

"Renaissance of Optical Microscopy" expresses the technical trend in the research of cell biology. The advanced studies of cell biology now requires the direct observation of the dynamics and function of living cells at the molecular level, and this necessity, coupled with the recent technological advances, such as laser technology, fast image processing, and the techniques for labeling specific molecules, is now driving the complete renovation of both hardwares and softwares for optical microscopy. I will review two newly developed techniques of optical microscopy in this presentation: (1) timeresolved microscope fluorimetry which was developed by us and (2) nanometer-video enhanced contrast microscopy developed by Sheetz et al. [1], and adapted by us recently.

(1) Time-resolved microscope fluorimetry. The excited-state lifetime of fluorescent molecules (about 1-20 nsec) located in a single living cell is measured under the microscope $(0.5 \mu \mathrm{m}$ spatial- and $20 \mathrm{psec}$ temporal-resolutions $)$. Proteinprotein association at the molecular level has been assessed by observing the resonance energy transfer (RET) from fluorescein (donor) to reactive red 8 (acceptor), both attached to IgG antibodies specific to, for example, desmoplakins, desmogleins, actin, and cadherin (RET decreases the lifetime of the donor). RET data suggested that cadherin forms molecular aggregates when they function as a cell adhesion molecule between two cells at the contacting regions.

(2) Nanometer-video enhanced contrast microscopy. The dynamics of individual receptor molecules (E-cadherin, transferrin receptor, a-macroglobulin receptor) is studied by tracking single colloidal gold particles of 20-40 nm diameter specifically attached to the receptor molecules via the monoclonal antibody or the ligand molecules (nanometer spatial and 30 msec temporal resolutions).

Diffusion of transferrin and $\alpha_{2}$-macroglobulin receptors is much faster than that of E-cadherin. However, for all these receptors, the mobility of individual molecules is limited within a certain small region in the membrane and they rarely go beyond the region. The diffusion coefficients inside the confined area are more than 10 times larger than those in the bulk region estimated previously by us by using the technique of fluorescence photobleaching recovery. These results support our proposed model, in which the plasma membrane is a patchwork of various domains.

[1] Sheetz, M. P., Tumey, S., Qian, H., \& Elson, E. Nature 340, 284-288 (1989). 